\title{
Scoping review: Diagnosis and management of periprosthetic joint infection in shoulder arthroplasty
}

\author{
Anthony Egglestone', Helen Ingoe', Jonathan Rees', \\ Michael Thomas ${ }^{3}$, Richard Jeavons ${ }^{4}$ and Amar Rangan ${ }^{1,5}$
}

\begin{abstract}
Background: The aim of this scoping review is to assess the current evidence regarding periprosthetic shoulder infection to inform development of evidence and consensus-based guidelines.

Methods: A search of Medline, Embase and PubMed was performed; two authors screened the results independently for inclusion.

Results: Totally 88 studies were included. Incidence of periprosthetic shoulder infection ranged from $0.7 \%$ to $7 \%$. The most common organisms to cause periprosthetic shoulder infection were Propionibacterium acnes, Staphylococcus aureus, Staphylococcus epidermidis and coagulase-negative Staphylococcus. Male gender and younger age are the most reported risk factors. Erythrocyte sedimentation rate, C-reactive protein and serum/synovial biomarkers had limited diagnostic accuracy. Thirty-nine studies reported the outcome of surgical management of periprosthetic shoulder infection. Eradication rates vary from $54 \%$ to $100 \%$ for debridement procedures; $66-100 \%$ for permanent spacers; $50-100 \%$ following single-stage revision; 60-100\% following two-stage revision; and 66-100\% following resection arthroplasty. Conclusion: There is wide heterogeneity in study designs and outcomes of studies are often contradictory and due to issues with methodology and small sample sizes the optimal pathways for diagnosis and management cannot be determined from this review. Future research should be based on larger cohorts and randomised trials where feasible to provide more valid research for guiding future treatment of periprosthetic shoulder infection.
\end{abstract}

\section{Keywords}

shoulder arthroplasty, shoulder replacement, infection, periprosthetic infection, diagnosis and management

Date received: IIth February 20I8; accepted: 30th April 2018

\section{Introduction}

Shoulder arthroplasty is an effective intervention for patients with painful shoulder arthritis. ${ }^{1}$ Although a rare complication, periprosthetic shoulder infection (PSI) can be devastating. In an attempt to cure or suppress infection patients could require multiple surgical procedures and prolonged treatment with antibiotics. ${ }^{2}$ The National Joint Registry (NJR) recorded 23,608 shoulder arthroplasty procedures between 2012 and 2016 and during this time 582 shoulders required revision (66 revised for infection). ${ }^{3}$

Much of the evidence pertaining to the diagnosis and management of periprosthetic joint infection (PJI) comes from the field of lower limb arthroplasty, which has led to the development of the
Musculoskeletal Infection Society (MSIS) diagnostic criteria. ${ }^{4}$ These criteria have been adopted for general diagnosis of PJI affecting most joints.

\footnotetext{
'Trauma and Orthopaedic Department, James Cook University Hospital, Middlesbrough, UK

${ }^{2}$ Nuffield Department of Orthopaedic Surgery, Nuffield Orthopaedic Centre, Oxford, UK

${ }^{3}$ Heatherwood and Wexham Park Hospitals Trust, Berkshire, UK

${ }^{4}$ North Tees and Hartlepool NHS Foundation Trust, Stockton on Tees, UK

${ }^{5}$ Faculty of Medical Sciences \& NDORMS, University of Oxford, Oxford, UK
}

\section{Corresponding author:}

Amar Rangan, Faculty of Medical Sciences \& NDORMS, University of Oxford, Oxford, UK.

Email: amar.rangan@york.ac.uk 
There is limited evidence examining the economic burden of PSI. Padegimas et al. reported the median cost of hospitalisation for treatment of infection to be around $\$ 17,163 .{ }^{5}$ Infection following lower limb arthroplasty has been more widely examined and Kurtz et al. found that hospitalisation costs of infected hips and knees to be 1.76 and 1.52 times higher, respectively, compared to uninfected implants, however this only accounted for the costs of inpatient care and not the total cost of treatment including rehabilitation and societal costs. ${ }^{6}$ A further study by Alp et al. found the cost of managing PJI in hip and knee replacements to range between 2 and 24 times higher than treating patients without infection. ${ }^{7}$

The aim of this scoping review is to assess the available evidence regarding incidence, risk factors, diagnosis and management of PSI to inform development of evidence and consensus-based guidelines by the British Elbow and Shoulder Society.

\section{Materials and methods}

\section{Search strategy}

A single search of Medline, Embase and PubMed was completed on 16 June 2017. There was no search of unpublished literature of trials' databases. Search strategies are shown in Figures 1, 2 and 3. No date restrictions were applied to the search but studies were restricted to English language.

\section{Study selection}

Studies were eligible for inclusion if they included data on incidence, risk factors, diagnosis or management of PSI. Studies' designs eligible for inclusion were systematic reviews, randomised controlled trials, cohort studies, case-control studies and case series of shoulder PJIs.

Case reports, expert opinions, letters to editor, articles relating to periprosthetic infections of joints other than the shoulder and literature reviews with no indication of systematic process were excluded.

Studies identified from the literature search were deduplicated and their titles and abstracts independently screened by two blinded authors (AE and $\mathrm{HI}$ ) using Rayyan software. ${ }^{8}$ Any potential studies for inclusion were submitted for full text search. The same two authors completed the full text search and any disagreements were resolved through discussion and if no agreement was reached the senior author (AR) arbitrated.

The Prisma diagram of the selection process can be found in Figure 4.

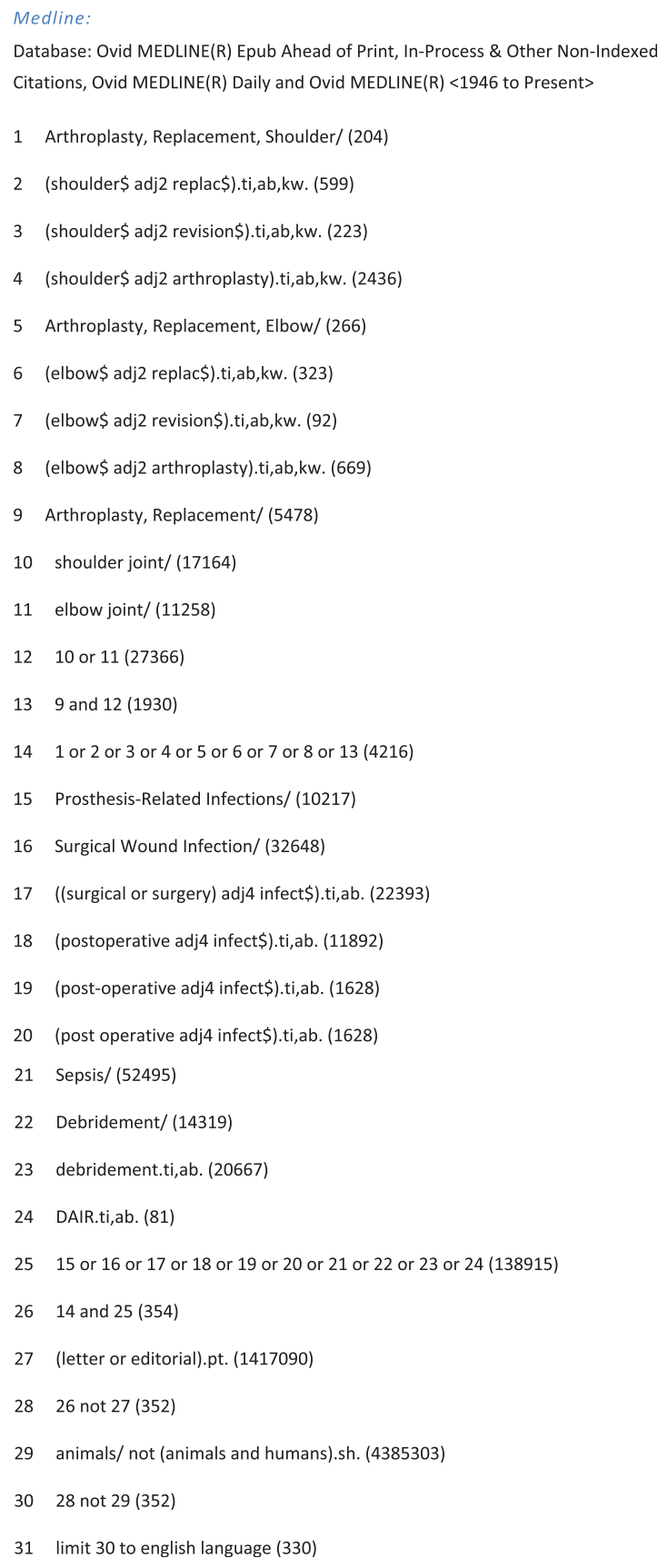

Figure I. Medline search strategy.

\section{Quality assessment}

The quality of papers was not formally evaluated using a validated assessment tool for risk of bias in this scoping review.

\section{Data extraction}

Data extraction was performed by one author (AE) and data was inserted into Supplementary Tables 1 to 8 . 


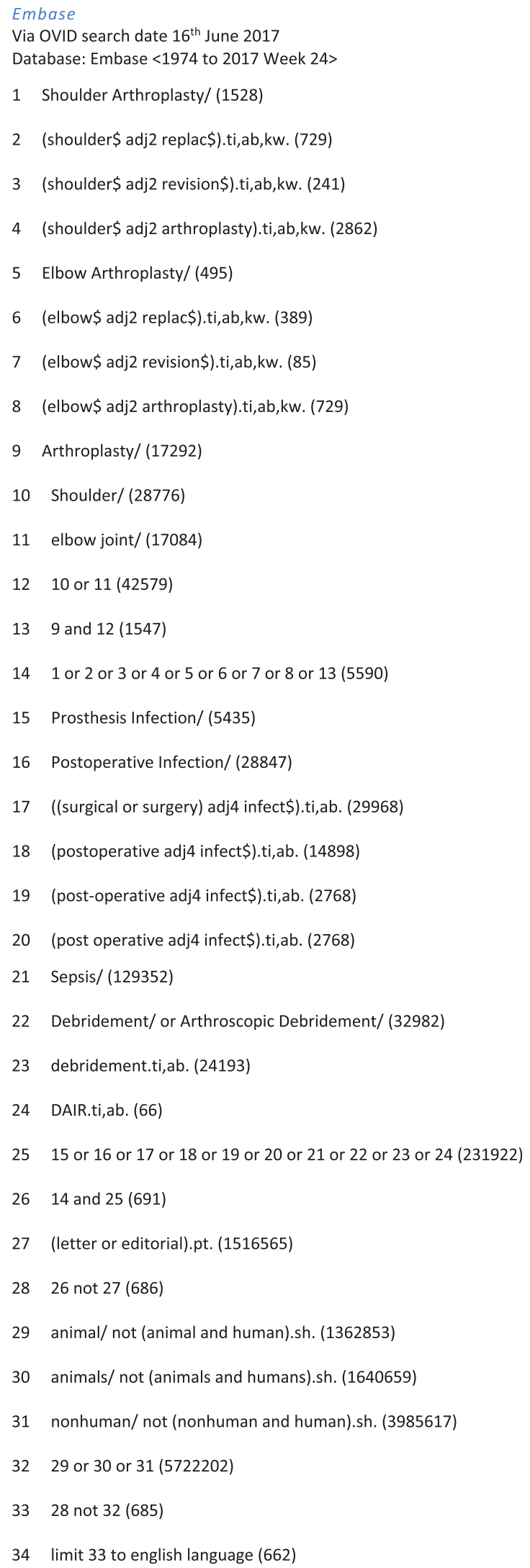

Figure 2. Embase search strategy.
This was then checked by a second author (HI). Data extracted from each study included: year of publication, study design, number of participants, methodology and results.

\section{Data synthesis}

A narrative synthesis was undertaken of the incidence, microbiological profile, risk factors, prophylactic measures against infection, diagnostic investigations and surgical management. The aim of the review was not to assess the effectiveness of diagnostic or treatment methods so pooling of data for meta-analysis was not performed.

\section{Results}

\section{Study characteristics}

The literature search of Embase, Medline and PubMed returned 891 unique studies. Of these, 141 studies were selected at initial screening and following full text screening 88 studies were eligible for inclusion in the final review. This consisted of four systematic reviews, ${ }^{9-12} 44$ retrospective case series, ${ }^{13-56} 23$ retrospective cohort studies, ${ }^{57-79} 12$ diagnostic studies, ${ }^{80-91}$ two in-vitro studies, ${ }^{92,93}$ two epidemiological studies ${ }^{5,94}$ and one economic decision analysis. ${ }^{95}$ The characteristics of included studies are shown in Supplementary Table 1 and excluded studies including reasons for exclusion are in Supplementary Table 2.

\section{Incidence of PSI}

Fourteen studies reporting the incidence of PSI were included. This included one systematic review, ${ }^{12}$ two epidemiological studies, ${ }^{5,94}$ five retrospective case series $^{41,45,46,52,55}$ and six retrospective cohort studies. ${ }^{60,62,73,75,77,78}$ The characteristics of the included studies are shown in Supplementary Table 3. The infection rate was between $0.08 \%$ and $5 \%$ for patients undergoing primary shoulder arthroplasty. Revision arthroplasty was associated with higher rates of infection, up to $32 \% .{ }^{55,96}$

The studies included in our review had a wide variation in follow-up time and different diagnostic criteria were used to diagnose PSI. This makes the true incidence of PSI difficult to determine from the information available. Further large-scale studies using accepted diagnostic criteria (such as MSIS criteria ${ }^{4}$ ) would give a more valid indication of the incidence of PSI.

Conclusion. There is an infection rate of $0.7 \%$ to $3.29 \%$ following conventional total shoulder replacement ${ }^{41,46,59,60} 0.5 \%$ to $2.7 \%$ following shoulder hemiarthroplasty $;^{41,45,59,78}$ and up to $5 \%$ following reverse 


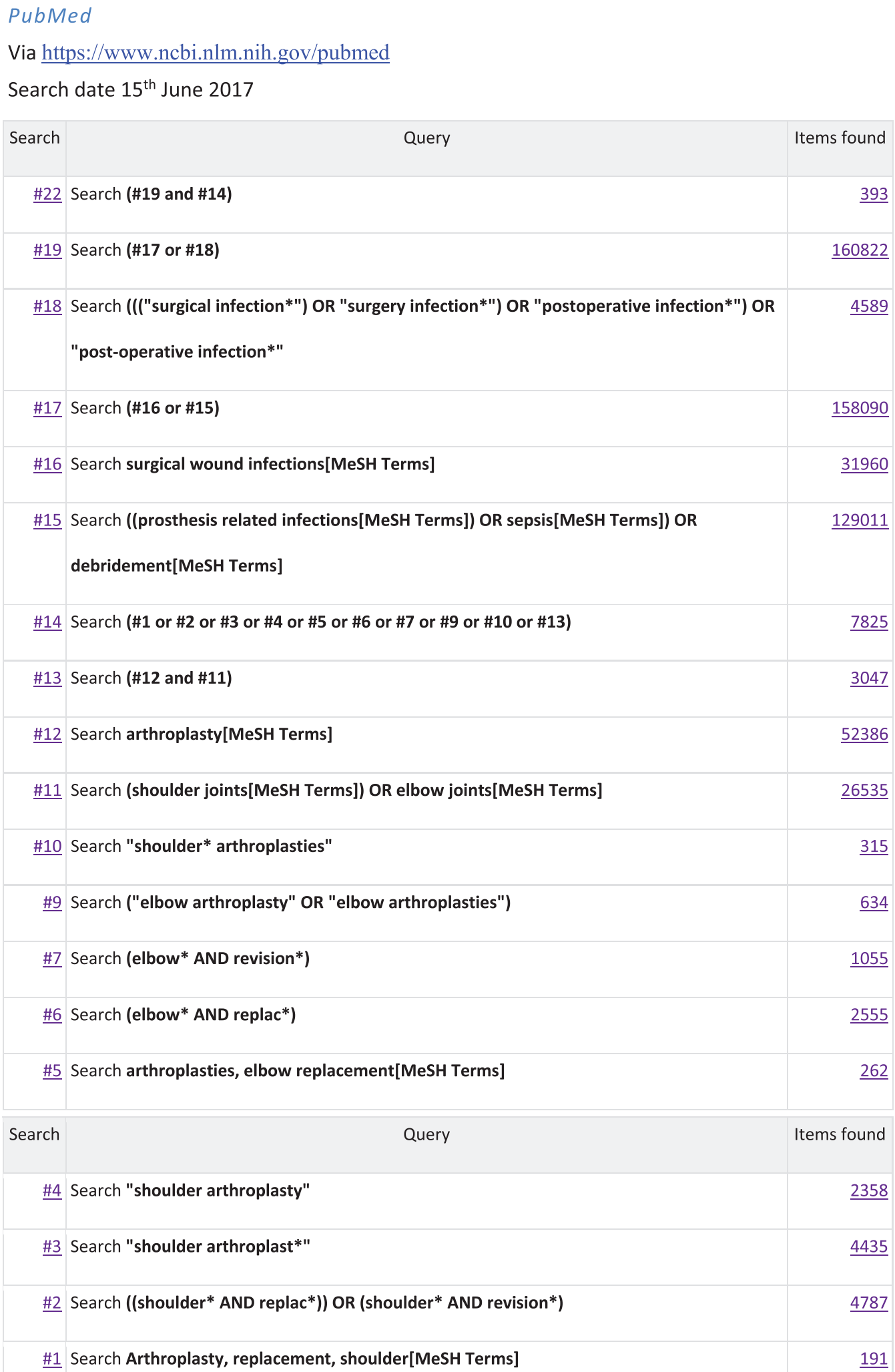

Figure 3. PubMed search strategy. 


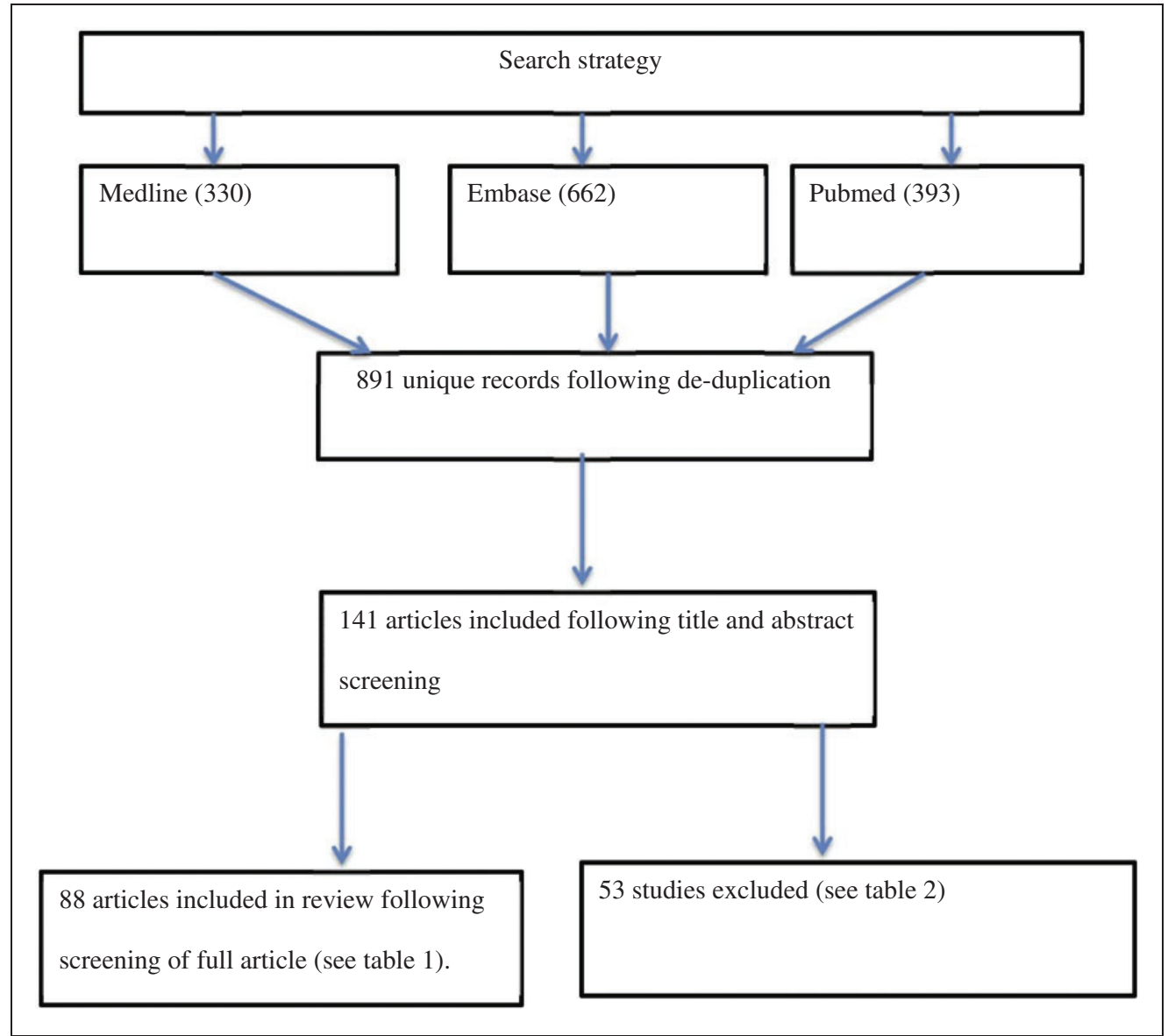

Figure 4. Prisma diagram.

shoulder arthroplasty. ${ }^{12,41,60,97}$ Florschutz et al., ${ }^{60}$ Smucny et al. $^{94}$ and Richards et al. $^{41}$ all reported higher infection rates in patients undergoing reverse shoulder arthroplasty compared to anatomic total shoulder arthroplasty. Revision shoulder arthroplasty had a much higher risk of infection with rates of $7 \% 55$ to $32 \% .{ }^{96}$

\section{Microbiological profile}

Our search identified 36 studies reporting the microbiological profile of PSI. This consisted of nine retrospective cohort studies; ${ }^{58,60,64,69-72,74} 22$ retrospective case series, ${ }^{13,15,17-19,21-23,28,29,31-33,35,41,43,45-47,50,51,53}$ three diagnostic studies, ${ }^{87,89,91}$ and one systematic review ${ }^{11}$ (see Supplementary Table 4). Our search also identified one in-vitro study reporting the susceptibility of Propionibacterium acnes to different antibiotics. ${ }^{92}$

Table 1 shows the commonly cultured organisms in PSIs. Other pathogens less commonly isolated included
Table I. Causative organisms of PSI.

\begin{tabular}{|c|c|c|}
\hline Pathogen & $\begin{array}{l}\text { Number of } \\
\text { studies }\end{array}$ & $\begin{array}{l}\text { Median infection } \\
\text { rate (range) }\end{array}$ \\
\hline Propionibacterium acnes & 33 & $29 \%(4-64 \%)$ \\
\hline $\begin{array}{c}\text { Coagulase negative } \\
\text { Staphylococcus }\end{array}$ & 22 & $22 \%(3.7-57.1 \%)$ \\
\hline $\begin{array}{l}\text { Methicillin sensitive } \\
\text { S. aureus }\end{array}$ & 27 & $20 \%(5.9-50 \%)$ \\
\hline S. epidermidis & 17 & $20 \%(4.5-55 \%)$ \\
\hline $\begin{array}{l}\text { Methicillin resistant } \\
\text { S. aureus }\end{array}$ & 16 & $8.7 \%(3.7-37.5 \%)$ \\
\hline Enterococcus sp. & 13 & $7 \%(2-12 \%)$ \\
\hline Streptococcus sp. & 13 & $6 \%(4.5-20 \%)$ \\
\hline Pseudomonas sp. & 10 & $5 \%(2-7 \%)$ \\
\hline
\end{tabular}


Corynebacterium, ${ }^{13,19,21,32,41,50,61,71,72,74,89,98}$ Neisseria sp., ${ }^{47,51}$ Clostridium sp., ${ }^{45,51,61,91}$ Klebsiella sp. ${ }^{21,50,98}$ and Mycobacterium. . $^{29,31,74}$

P. acnes is one of the common bacteria to cause PSI. One in-vitro study showed that penicillin G, cephalothin and ceftriaxone had the lowest minimum inhibitory concentrations (MIC) against P. acnes. ${ }^{92}$

Conclusion. Our searches identified one systematic review $^{11}$ which groups 26 studies (629 patients) and reported the average infection rates of different pathogens. $P$. acnes was the most common bacterium (38.9\%) followed by Staphylococcus aureus (14.8\%), Staphylococcus epidermidis $(14.5 \%)$ and coagulase negative Staphylococcus (14\%).

\section{Risk factors for PSI}

Our search identified 16 studies reporting risk factors for PSI. This included one systematic review, ${ }^{12}$ six retrospective cohort studies, ${ }^{57,60,62,69,77,78}$ seven retrospective case series ${ }^{14,20,41,45,46,52,54}$ and two epidemiological studies. ${ }^{5,94}$ The characteristics of included studies can be found in Supplementary Table 5.

The included primary studies had different diagnostic criteria for PSI, follow-up times and numbers of participants. Due to this heterogeneity it is difficult to draw valid conclusions regarding risk factors for PSI.

Four studies reported significantly higher risk of PSI in males ${ }^{5,41,46,69}$ and four studies reported higher infection rates in patients aged under $65 .{ }^{5,41,46,59}$ Five studies reported risk of PSI associated with different comorbidities. ${ }^{5,54,57,69,94}$ Smucny et al. reported higher infection rates associated with heart failure, peripheral vascular disease, chronic lung disease, diabetes mellitus, liver disease, renal failure and coagulopathy. ${ }^{94}$ Rheumatoid arthritis and patients on long-term steroids had increased risk of infection in one study ${ }^{59}$ and malnutrition, drug abuse and anaemia were found to be risk factors in another. ${ }^{5}$ Smoking status was also associated with a significantly increased risk of infection. ${ }^{62}$ Obesity was associated with significantly increased infection rates in one study ${ }^{94}$ and a non-significant risk increase another. ${ }^{45}$ Anakwenze et al. found that increased body mass index was associated with higher infection rates following reverse shoulder arthroplasty but not in conventional shoulder replacement. ${ }^{57}$ Morris et al. found no association between obesity and PSI. ${ }^{69}$ One study found that HIV patients had a significantly higher risk of PSI at 90 days $(p<0.01)$ and at two years $(p=0.04) .^{54}$

Two studies found revision arthroplasty to have significantly higher infection rates than primary arthroplasty. ${ }^{59,69}$ Reverse shoulder arthroplasty was also associated with higher infection rates when compared to conventional shoulder arthroplasty. ${ }^{12,41}$ Arthroplasty for trauma ${ }^{41,94}$ and patients requiring arthroplasty for cuff tear arthropathy or avascular necrosis ${ }^{94}$ also had increased risk of PSI.

Other factors associated with increased infection risk include blood transfusion; $;^{59,94}$ haematoma formation requiring evacuation; ${ }^{52}$ length of hospital stay and patients requiring mechanical ventilation. ${ }^{94}$ Walch et al. found a trend towards lower infection rates with increased surgeon experience but this did not reach statistical significance. $^{75}$

Previous treatments such as steroid injections within three months of arthroplasty and patients having previous non-arthroplasty surgery on the shoulder were shown to increase the risk of PSI in three studies. ${ }^{59,77,78}$

In contrast, other studies showed no increased infection risk with: gender, ${ }^{45}$ age, ${ }^{45}$ cormorbidities, ${ }^{45,46}$ smoking status, ${ }^{69}$ diagnosis for procedure, ${ }^{46}$ type of implant used ${ }^{60}$ previous non-arthroplasty surgery ${ }^{69}$ or arthroplasty for trauma. ${ }^{69}$

Evidence from the current literature is therefore not consistent, which may be a reflection of the low event rate with periprosthetic infection following shoulder arthroplasty, mixed populations within these studies or under reporting of such complications. Future research with pooled data for meta-analysis and use of shoulder replacement registry data would help further clarify risk factors and associations for periprosthetic shoulder joint infections.

Conclusion. The body of current evidence however suggests that males and patients under 65 have a higher risk of PSI. Alongside this there is some evidence to suggest that previous treatments, indications for arthroplasty, patient comorbidities (especially diabetes, smoking and obesity) and surgical factors (operating time and complications) are all linked to higher risk of PSI.

\section{Prophylaxis against PSI}

Our search identified nine studies that reported on techniques for prophylaxis against PSI. This included four retrospective case series, ${ }^{25,36,38,40}$ four retrospective cohort studies ${ }^{66-68,70}$ and one economic decision analysis $^{95}$ (see Supplementary Table 6).

Five studies reported the presence of Propionibacterium on intraoperative cultures despite preoperative intravenous antibiotics and skin preparation. ${ }^{25,36,38,40,68}$ Studies by Levy et al., Matsen et al. and Phandis et al. all found growth of $P$. acnes on intraoperative cultures despite preoperative i.v. antibiotics and skin preparation. ${ }^{36,38,40}$ Maccioni et al. used the Oxford technique (whereby separate sterile instruments are used for each sample) for collecting intraoperative samples and patients received $2 \mathrm{~g}$ cefazolin peri-operatively with Povoidone skin prep and Ioban draping. ${ }^{68}$ This resulted in a $3.125 \%$ positive 
culture rate and suggests that the high rates of $P$. acnes in previous studies may have been due to collection technique.

Falconer et al. tried to identify the potential sources of contamination in shoulder arthroplasty and found that despite $2 \mathrm{~g}$ cefazolin and $10 \%$ povidone-iodine solution with $30 \%$ ethylalchol and ioban that $40 \%$ of subdermal swabs were positive for P. acnes. ${ }^{25}$ This study also found $17.5 \%$ of forceps and surgeon's gloves grew $P$. acnes, $10 \%$ of skin blades and $2.5 \%$ of inside blades grew P. acnes. ${ }^{25}$

These studies all showed deficiencies with standard methods of prophylaxis. Lorenzetti et al. showed that by using a cyanoacrylate microbial sealant alongside standard preparation with i.v. antibiotics, chloraprep and Ioban that positive culture rate for $P$. acnes reduced from $18 \%$ to $7 \%{ }^{66}$

One study showed significant reduction in PSI when antibiotic impregnated bone cement was used as opposed to plain bone cement. ${ }^{70}$ A further study found that injecting the glenohumeral joint with gentamycin at the end of surgery also significantly reduced the PSI rate. ${ }^{67}$

Hatch et al. calculated that if vancomycin powder placed into the operative wound resulted in a $0.01 \%$ to $0.19 \%$ absolute risk reduction for PSI then it would be cost effective (depending on the purchase prices of vancomycin at different centres). ${ }^{95}$ Our search has not identified any studies demonstrating the effectiveness of vancomycin power in shoulder arthroplasty.

Conclusion. The current body of evidence suggests that antibiotic impregnated bone cement and intraarticular gentamycin injections can result in a statistically significant reduction in PSI rates, however these are findings from individual small studies. Current i.v. antibiotic and skin preparation regimes may be insufficient at eradicating possible pathogens in the surgical field.

Further studies comparing chloraprep, povidoneiodine and other antiseptic skin solutions for efficacy at eradicating not only $P$. acnes but also other pathogens should be considered. The studies showing benefit of antibiotic bone cement and intra-articular antibiotic injections were both retrospective cohort studies and should be backed up with prospective studies, ideally randomised trials.

\section{Diagnosis}

Our search identified two diagnostic criteria for PJI. The MSIS defined infection as being present when one of the criteria in Figure 5 is present. ${ }^{4}$ Frangiamore et al. devised a separate classification for PSI as shown in Figure $6 .^{82}$

Our search identified four studies evaluating the diagnostic accuracy of C-reactive protein (CRP) and erythrocyte sedimentation rate $(\mathrm{ESR})^{82,85,88,91}$ (see Table 2). The sensitivity of CRP ranged from $0 \%{ }^{91}$ to $46 \%{ }^{85}$ and its specificity ranged from $84 \%{ }^{88}$ to $95 \% .{ }^{91}$ ESR had a sensitivity ranging from $16 \%{ }^{88}$ to $42 \%{ }^{85}$ and specificity of $65 \%{ }^{91}$ to $98 \%{ }^{88}$ These studies demonstrate ESR and CRP are of limited use in the diagnosis of PJI.

Six prospective diagnostic studies investigating the diagnostic utility of novel biomarkers were included $^{81-83,85,87,91}$ (see Supplementary Table 7). Two studies $^{85,91}$ reported the diagnostic accuracy of serum interleukin-6 (IL-6) and a further study reported the accuracy of synovial IL- $6^{83}$ for diagnosis of PSI. The sensitivity and specificity of serum IL-6 were $12.5-14 \%$ and $93-95 \%$, respectively ${ }^{91,85}$ suggesting this is a poor marker for identifying infection. Synovial IL-6 was found to be more accurate for infection with sensitivity and specificity of $87 \%$ and $90 \%$, respectively. ${ }^{83}$

Frangiamore et al. examined the utility of a range of synovial cytokines in one study. They found that a combined profile of synovial IL-6, TNF-alpha and IL-2 had the best predictive power for infection with a sensitivity of $80 \%$ and specificity of $93 \% .{ }^{81}$

Alpha-defensin was found to have a sensitivity of $78 \%$ and specificity of $63 \%$ for diagnosing $\mathrm{PSI}^{82}$ and synovial leukocyte esterase was found to have limited use with a sensitivity of $30 \%$ and specificity of $67 \% .{ }^{87}$

Our search identified two papers that examined the role of pre-revision biopsy to diagnose PSI. Dilisio et al. found arthroscopic biopsy had a sensitivity and specificity of $100 \%$ when compared to intraoperative samples in their series of 19 patients with chronic infection (time between index procedure and arthroplasty of $3.0 \pm 2.0$ years). In comparison fluoroscopic guided aspiration yielded sensitivity and specificity of $16.7 \%$ and $100 \%$, respectively. ${ }^{80}$ Tashjian et al. showed that pre-revision biopsy had a sensitivity of $90 \%$ and specificity of $86 \% .^{90}$

The diagnosis of $P$. acnes can often be challenging due to the insidious nature of the infection and samples often require longer incubation periods to confirm positive cultures. Two studies reported the incubation time required to detect $P$. acnes on culture. Dodson et al. showed that the average time from sampling to positive cultures was nine days ${ }^{24}$ whereas Wang et al. detected $100 \%$ of $P$. acnes cases by day $5 .^{51}$ These were small studies of 11 and 17 cases and further reports with larger samples for the range of common pathogens would be beneficial. Nodzo et al. found that haemolytic strains of $P$. acnes were more likely to be associated with true infection rather than contamination. ${ }^{93}$

Conclusion. Evidence from the literature is inconsistent and the trials identified are hampered by low sample sizes resulting in underpowered studies. Further primary studies with appropriate power or meta-analyses 
1. There is a sinus tract communicating with the prosthesis; or

2. A pathogen is isolated by culture from at least two separate tissue or fluid samples obtained from the affected prosthetic joint; or

3. Four of the following six criteria exist

- Elevated serum erythrocyte sedimentation rate (ESR) and serum C-reactive protein (CRP) concentration

- Elevated synovial leukocyte count

- Elevated synovial neutrophil percentage

- Presence of purulence in the affected joint

- Isolation of a microorganism in one culture of periprosthetic tissue or fluid

- Greater than five neutrophils per high-power field in five high-power fields observed from histologic analysis of periprosthetic tissue at $\mathrm{x} 400$ magnification

Figure 5. MSIS diagnostic criteria.

of current studies are required to identify the true sensitivity and specificity of ESR/CRP and the novel biomarkers of infection. Pre-revision biopsy has shown promising results in small trials for identification of responsible organisms; this needs to be balanced against the risks of a more invasive procedure and further large-scale trials are required to confirm its utility for PSI diagnosis.

\section{Surgical management of PSI}

Our search identified 39 studies reporting on the surgical management of PSI. This included three systematic reviews, ${ }^{9-11} 27$ retrospective case series $^{13,15-19,21-24,27-31,34,35,37,42-44,47-50,53,56}$ and nine retrospective cohort studies ${ }^{58,61,64,65,71,72,74,76,96}$ (see Supplementary Table 8).

Implant retention procedures. Our search identified 12 primary studies and two systematic reviews ${ }^{10,11}$ reporting the outcome in patients undergoing irrigation and debridement procedures. The primary research included five retrospective case series ${ }^{13,15,23,42,53}$ and seven retrospective cohort studies. ${ }^{58,61,64,65,71-73}$

The success rate for treating acute infection using debridement and implant retention (DAIR) procedures ranged from $54 \%{ }^{64}$ to $100 \% .{ }^{61,65}$ Study size ranged from one ${ }^{61}$ through to 13 patients ${ }^{64}$ and due to the small sizes it is difficult to draw valid conclusions. 


\section{Definite infection}

- At least one positive pre-operative or intra-operative finding of infection and multiple positive intra-operative cultures with the same organism

OR

- One positive pre-operative (aspirate) culture and one positive intra-operative culture with the same organism

\section{Probable infection}

- At least one positive pre-operative or intra-operative finding of infection and one positive intra- operative culture

OR

- No pre-operative or intra-operative findings of infection and multiple positive intra-operative cultures with the same organism

\section{Probable contaminant}

- No pre-operative or intra-operative findings of infection and one positive intraoperative culture

\section{No evidence for infection}

- No pre-operative or intra-operative findings of infection and no positive intraoperative cultures

Figure 6. Frangiamore classification.

Zavala et al. reported outcomes of five acute infections and two sub-acute infections following reverse arthroplasty treated with debridement procedures. In three of the patients, humeral polyethylene exchange was performed which resulted in clearance of infection. Of the remaining patients in which no humeral polyethylene exchange was performed two required further resection arthroplasty. ${ }^{25}$ Jacquot et al. found that debridement procedures with exchange of humeral polyethylene component resulted in only $44 \%$ eradication rate. This was increased to $75 \%$ when humeral polyethylene exchange was combined with exchange of glenosphere. ${ }^{41}$

Two systematic reviews reported $69 \%$ and $70 \%$ eradication rates following DAIR procedures. ${ }^{10,11}$

The time between the index procedure and the presentation of infection can affect the potential success of debridement procedures. Zavala et al. found a $100 \%$ eradication rate when patients had debridement procedure within two weeks but success rates of only $33 \%$ when debridement was done two months after the index procedure. ${ }^{53}$ This was only an extremely 
Table 2. Diagnostic accuracy of CRP and ESR.

\begin{tabular}{|lcc|}
\hline Study & Sensitivity & Specificity \\
\hline Frangiamore $^{82,83}$ & & \\
\hline CRP & 46 & 96 \\
\hline ESR & 42 & 82 \\
\hline Grosso et al. ${ }^{84,85}$ & & \\
\hline CRP & 46 & 93 \\
\hline ESR & 42 & 82 \\
\hline Piper et al. ${ }^{88}$ & & 84 \\
\hline CRP & 42 & 98 \\
\hline ESR & 16 & \\
\hline Villacis et al. ${ }^{91}$ & & 95 \\
\hline CRP & 0 & 65 \\
\hline ESR & 21 & \\
\hline
\end{tabular}

small series of seven patients and further larger scale studies, if conducted, would help identify more clearly the time point beyond which debridement procedures are less likely to be successful.

Resection with permanent cement spacer. Seventeen studies reporting the outcomes of resection and implantation of antibiotic loaded cement spacer were included. This included three systematic reviews, ${ }^{9-11}$ six retrospective cohort studies $58,61,64,71,72,74$ and eight retrospective case series. $^{27,32,35,37,47,49,50,56}$ Primary research studies had populations of seven to 32 patients. The eradication rate following permanent cement spacer was $66 \%{ }^{64}$ $100 \%{ }^{21,27,35,37,47,49,50,58,61,71,74}$ Function was most commonly assessed using the Constant score which ranged from $26^{74}$ to $57 .^{21}$

Three systematic reviews reported the average eradication rates between $90 \%$ and $95.6 \%{ }^{9-11}$ with average constant scores of 31-32 in the third. ${ }^{9-11}$

Single-stage revision. Our search identified 14 studies that reported outcomes of single-stage revision for PSI. There were seven retrospective case series, ${ }^{15,17,22,28,30,31,34}$ three retrospective cohort studies, one diagnostic study $^{26}$ and three systematic reviews. $^{9-11,58,64,73}$ Primary research studies had populations ranging from two ${ }^{96}$ patients to 55 patients. ${ }^{30}$ Eradication rates from these studies ranged from $50 \%{ }^{6}$ to $100 \% .^{22,30,31,58,64}$ The post op Constant score ranged from 33 to 66 . Cuff et al. noted no significant difference in American Shoulder and Elbow Score or visual analogue pain score between patients undergoing one and two stage revision. ${ }^{22}$

The three systematic reviews reported infection clearance rates of $92 \%-97 \%$ with postoperative Constant scores averaging 48.1 to $51 .^{9-11}$

Grosso et al. reported on 17 patients undergoing single-stage revision with unexpected positive cultures. Only $5.7 \%$ of patients with positive cultures developed a PJI at final follow up. Foruria et al. ${ }^{26}$ found a prevalence of $15 \%$ for unexpected positive cultures following revision shoulder arthroplasty with most cases being due to $P$. acnes. Of these $10 \%$ developed PJI caused by the same organism. ${ }^{26}$

Conclusion. From the literature identified we are unable to comment on what variables are more likely to produce a successful outcome following single-stage revision. Further research identifying patient factors (such as co-morbidities and soft tissue status), microbiological factors (such as sensitivity of underlying organism) and surgical factors (such as surgeon experience and exchange of polyethylene/glenosphere components) associated with successful outcome of single-stage revision would help guide future surgical treatment. Further studies investigating follow-up and management of patients with unexpected positive cultures would be valuable to guide appropriate management in this cohort of patients.

Two-stage revision. We identified 17 primary research papers published reporting the outcomes of two stage revision - 10 retrospective case series $^{13,15,16,19,22,29,43,44,48,79}$ and seven retrospective cohort studies. ${ }^{58,61,64,65,71-73}$ Primary studies had populations of three ${ }^{96}$ to 36 cases. ${ }^{16}$ Successful treatment of infection was reported from $60 \%^{58}$ to $100 \%$ of cases. ${ }^{44,61,65,72,96}$ The three systematic reviews reported successful outcomes of $86 \%,{ }^{10} 91 \%{ }^{9}$ and $94 \%{ }^{11}$ for eradication of infection.

Functional assessment was carried out using Constant-Murley scoring for seven studies with results ranging from $22.7^{61}$ to $52.2 .{ }^{71}$ Neer's score was used to determine the outcome in Assenmacher's series (28\% excellent and $33 \%$ satisfactory outcome) $)^{16}$ and Stickland's series $(10 \%$ excellent and $20 \%$ satisfactory). ${ }^{48}$

Following implantation of cement spacer all papers reported that patients were started on an antimicrobial regime in liaison with microbiology/infectious disease specialists and replantation of definite prosthesis was planned for when infection was deemed eradicated by using combination of clinical examination, radiographic findings and inflammatory markers. The average times between first and second stage revision ranged from 2.5 months $^{19}$ to 6.6 months. $^{29}$ 
Zhang et al. performed open biopsy following six weeks of i.v. antibiotics to detect persistent infection prior to replantation. Four of their 18 patients had positive biopsy results and required a further round of irrigation and debridement followed by antibiotics prior to definitive revision. They report that no patients have developed recurrent infection following second-stage revision. ${ }^{79}$

Conclusion. From current literature, the optimum length of antibiotic treatment between stages; the most appropriate class of empirical antibiotic treatment; or the most accurate method of ensuring infection eradication prior to implantation of revision prosthesis remain uncertain and further research aimed at answering these questions would be useful.

Resection arthroplasty. Our search identified 10 primary research studies and three systematic reviews reporting outcomes following resection arthroplasty for PSI. The primary research studies consisted of two retrospective case series ${ }^{18,53}$ and eight retrospective cohort studies. ${ }^{58,61,64,71-74,76}$ The retrospective case series had sample sizes of one to seven patients. The seven retrospective cohort studies ${ }^{18,58,61,64,71,74,96}$ had samples of $1-21$ patients. Eradication was achieved in $66 \%{ }^{64}$ to $100 \%{ }^{18,71}$ of cases. The three systematic reviews reported eradication as averaging $84 \%$ to $89 \%$.

Function was reported using Constant-Murley score in seven of the primary studies with results ranging from 17 to $38 .^{58,61,64,71,72,74,76}$ The three systematic reviews reported average functional outcome using Constant score as $29,{ }^{10} 32^{9}$ and 33.5. ${ }^{11}$

Conclusion. From the current literature, resection arthroplasty can offer a suitable salvage however there is a wide range of eradication rates and patient outcomes. Further research is required to identify which patients will get best outcomes from the procedure.

\section{Conclusion}

The outcomes reported from the current published literature show very little difference in outcome between management options regards to eradication of infection and functional outcome for patients. Due to the rarity of PSI the numbers reported are too small to draw valid conclusions. There is a need for further larger scale studies to compare the outcomes of these procedures.

The decision on which surgical intervention is used for which patient is based on the clinical experience of the surgeons supplemented by discussion with patients. We found little reasoning in the literature explaining why patients are treated with one technique over another and further research is required to identify which patients would benefit most from resection or revision (either permanent spacer, one stage or two stage). Variables such as the length of time between the stages of a two-stage revision or the length of postoperative antibiotic therapy following definitive surgery are poorly reported in the literature. Further research should be conducted to evaluate the appropriate postoperative antimicrobial regimes including length of intravenous and oral antibiotic regimes and the most sensitive monitoring regimes to detect when infection has been eradicated.

\section{Discussion}

Clinical diagnosis of PJI can be difficult, as low-grade infections may present with non-specific and vague symptoms such as pain and stiffness. Clinical history and examination, inflammatory markers and shoulder aspiration are often the first line investigations for suspected periprosthetic infection. The current research shows that this approach may be of limited use due to the low sensitivity and specificity of this approach. There is a role for further research focusing on the diagnostic accuracy of novel biomarkers of infection and the role of pre-revision biopsy.

Current evidence regarding surgical management of periprosthetic infection is also contradictory and further research is required to identify which patients are more likely to benefit from the different surgical options. There is also further research needed to look at the factors associated with positive outcome following revision surgery including:

- Patient factors: comorbidities, soft tissue status, socioeconomic status

- Microbiological factors: organisms involved, resistance patterns

- Treatment factors: surgeon and centre volume, length and class of antibiotic treatment, staging and type of revision procedure

Our search strategy only captured published studies on shoulder PJI and not the grey literature or narrative literature reviews. We have not reviewed data from lower limb PJI, which may be seen as a potential limitation, but to what extent that knowledge is applicable to shoulder PJI remains uncertain. Further research to explore the findings regarding diagnosis and management of lower limb PJI ${ }^{100-102}$ and there relevance to shoulder infection would be beneficial.

The current body of literature is limited and mainly based on small retrospective case series and cohort studies with few registry studies. Future research should be based on larger cohorts, randomised trials where feasible and potentially large registry data studies to provide more reliable research for guiding future treatment of PSI. 


\section{Declaration of Conflicting Interests}

The author(s) declared no potential conflicts of interest with respect to the research, authorship, and/or publication of this article.

\section{Funding}

The author(s) received no financial support for the research, authorship, and/or publication of this article.

\section{Supplementary material}

Supplementary material is available at journals.sagepub.com/ doi/suppl/10.1177/1758573218779076.

Supplementary Table 1. Included studies.

Supplementary Table 2. Excluded studies.

Supplementary Table 3. Incidence of PSI.

Supplementary Table 4. Microbiological profile.

Supplementary Table 5. Risk factors for PSI.

Supplementary Table 6. Prophylaxis.

Supplementary Table 7. Diagnostic studies.

Supplementary Table 8. Surgical management of PSI.

\section{References}

1. Franceschini V and Chillemi C. Periprosthetic shoulder infection. Open Orthop J 2013; 7: 243-249.

2. Seitz WH. Shoulder-joint infection: diagnosis and management. Semin Arthroplasty 2011; 22: 42-47.

3. National Joint Registry for England W, Northern Ireland and Isle of Man. 13th Annual Report. 2016.

4. Parvizi J, Zmistowski B, Berbari EF, et al. New definition for periprosthetic joint infection: from the workgroup of the Musculoskeletal Infection Society. Clin Orthop Rel Res 2011; 469: 2992-2994.

5. Padegimas EM, Maltenfort M, Ramsey ML, et al. Periprosthetic shoulder infection in the United States: incidence and economic burden. J Shoulder Elbow Surg 2015; 24: 741-746.

6. Kurtz SM, Lau E, Watson H, et al. Economic burden of periprosthetic joint infection in the United States. $J$ Arthroplasty 2012; $27(8$ suppl): 61-5.e1.

7. Alp E, Guney A, Cevahir F, et al. Incidence and economic burden of prosthetic joint infections in a university hospital: a report from a middle-income country. J Infect Public Health 2016; 9: 494-498.

8. Ouzzani M, Hammady H, Fedorowicz Z, et al. Rayyan a web and mobile app for systematic reviews. Syst Rev 2016; 5 .

9. George DA, Volpin A, Scarponi S, et al. Does exchange arthroplasty of an infected shoulder prosthesis provide better eradication rate and better functional outcome, compared to a permanent spacer or resection arthroplasty? a systematic review orthopedics and biomechanics. BMC Musculoskelet Disord 2016; 17: 52.

10. Marcheggiani Muccioli GM, Huri G, Grassi A, et al. Surgical treatment of infected shoulder arthroplasty. A systematic review. Intl Orthop 2017; 41: 823-830.

11. Nelson GN, Davis DE and Namdari S. Outcomes in the treatment of periprosthetic joint infection after shoulder arthroplasty: a systematic review. J Shoulder Elbow Surg 2016; 25: 1337-1345.

12. Zumstein MA, Pinedo M, Old J, et al. Problems, complications, reoperations, and revisions in reverse total shoulder arthroplasty: a systematic review. J Shoulder Elbow Surg 2011; 20: 146-157.

13. Achermann Y, Sahin F, Schwyzer $H$, et al. Characteristics and outcome of 16 periprosthetic shoulder joint infections. Infection 2013; 41: 613-620.

14. Aibinder WR, Schoch BS, Cofield RH, et al. Safety and efficacy of shoulder arthroplasty following lower extremity periprosthetic joint infection. J Shoulder Elbow Surg 2017; 26: 79-84.

15. Amaravathi RS, Kany J, Melet M, et al. Analysis of infection in shoulder arthroplasty: a multicentre study. Europ J Orthop Surg Traumatol 2012; 22: 145-150.

16. Assenmacher AT, Alentorn-Geli E, Sanchez-Sotelo J, et al. Two-stage reimplantation for the treatment of deep infection after shoulder arthroplasty. J Shoulder Elbow Surg 2005; 26: e163.

17. Beekman PDA, Katusic D, Berghs BM, et al. One-stage revision for patients with a chronically infected reverse total shoulder replacement. J Bone Joint Surg Ser B 2010; 92: 817-822.

18. Braman JP, Sprague M, Bishop J, et al. The outcome of resection shoulder arthroplasty for recalcitrant shoulder infections. J Shoulder Elbow Surg 2006; 15: 549-553.

19. Buchalter DB, Mahure SA, Mollon B, et al. Two-stage revision for infected shoulder arthroplasty. J Shoulder Elbow Surg 2017; 26: 939-947.

20. Cheung EV, Sperling JW and Cofield RH. Infection associated with hematoma formation after shoulder arthroplasty. Clin Orthop Relat Res 2008; 466: 1363-1367.

21. Coffey MJ, Ely EE and Crosby LA. Treatment of glenohumeral sepsis with a commercially produced antibioticimpregnated cement spacer. J Shoulder Elbow Surg 2010; 19: 868-873.

22. Cuff DJ, Virani NA, Levy J, et al. The treatment of deep shoulder infection and glenohumeral instability with debridement, reverse shoulder arthroplasty and postoperative antibiotics. J Bone Joint Surg Ser B 2008; 90: 336-342.

23. Dennison T, Alentorn-Geli E, Assenmacher AT, et al. Management of acute or late hematogenous infection after shoulder arthroplasty with irrigation, debridement, and component retention. J Shoulder Elbow Surg 2017; 26: $73-78$.

24. Dodson CC, Craig EV, Cordasco FA, et al. Propionibacterium acnes infection after shoulder arthroplasty: a diagnostic challenge. J Shoulder Elbow Surg 2010; 19: 303-307.

25. Falconer TM, Baba M, Kruse LM, et al. Contamination of the surgical field with Propionibacterium acnes in primary shoulder arthroplasty. J Bone Joint Surg 2016; 98-A: 1722-1728.

26. Foruria AM, Fox TJ, Sperling JW, et al. Clinical meaning of unexpected positive cultures (UPC) in revision shoulder arthroplasty. J Shoulder Elbow Surg 2013; 22: $620-627$. 
27. Gorman MT and Crosby LA. Treatment of deep infection after total shoulder arthroplasty with an antibioticimpregnated cement spacer. Tech Shoulder Elbow Surg 2006; 7: 82-85.

28. Grosso MJ, Sabesan VJ, Ho JC, et al. Reinfection rates after 1-stage revision shoulder arthroplasty for patients with unexpected positive intraoperative cultures. J Shoulder Elbow Surg 2012; 21: 754-758.

29. Hattrup SJ and Renfree KJ. Two-stage shoulder reconstruction for active glenohumeral sepsis. Orthopedics 2010; 33: 20.

30. Hsu JE, Gorbaty JD, Whitney IJ, et al. Single-stage revision is effective for failed shoulder arthroplasty with positive cultures for propionibacterium. J Bone Joint Surg Am 2016; 98: 2047-2051.

31. Ince A, Seemann K, Frommelt L, et al. One-stage exchange shoulder arthroplasty for peri-prosthetic infection. J Bone Joint Surg Br 2005; 87: 814-818.

32. Jawa A, Shi L, O'Brien T, et al. Prosthesis of antibioticloaded acrylic cement (PROSTALAC) use for the treatment of infection after shoulder arthroplasty. $J$ Bone Joint Surg Ser A 2011; 93: 2001-2009.

33. Kelly IJD and Hobgood ER. Positive culture rate in revision shoulder arthroplasty. Clin Orthop Rel Res 2009; 467: 2343-2348.

34. Klatte TO, Junghans K, Al-Khateeb H, et al. Single-stage revision for peri-prosthetic shoulder infection: outcomes and results. Bone Joint J 2013; 95: 391-395.

35. Levy JC, Triplet $\mathbf{J}$ and Everding N. Use of a functional antibiotic spacer in treating infected shoulder arthroplasty. Orthopedics 2015; 38: e512-e519.

36. Levy O, Iyer S, Atoun E, et al. Propionibacterium acnes: an underestimated etiology in the pathogenesis of osteoarthritis? J Shoulder Elbow Surg 2013; 22: 505-511.

37. Mahure SA, Mollon B, Yu S, et al. Definitive treatment of infected shoulder arthroplasty with a cement spacer. Orthopedics 2016; 39: e924-e930.

38. Matsen FA, 3rd, Russ SM, Bertelsen A, et al. Propionibacterium can be isolated from deep cultures obtained at primary arthroplasty despite intravenous antimicrobial prophylaxis. J Shoulder Elbow Surg 2015; 24: 844-847.

39. McGoldrick E, McElvany MD, Butler-Wu S, et al. Substantial cultures of Propionibacterium can be found in apparently aseptic shoulders revised three years or more after the index arthroplasty. J Shoulder Elbow Surg 2015; 24: 31-35.

40. Phadnis J, Gordon D, Krishnan J, et al. Frequent isolation of Propionibacterium acnes from the shoulder dermis despite skin preparation and prophylactic antibiotics. J Shoulder Elbow Surg 2016; 25: 304-310.

41. Richards J, Inacio MCS, Beckett M, et al. Patient and procedure-specific risk factors for deep infection after primary shoulder arthroplasty. Clin Orthop Rel Res 2014; 472: 2809-2815.

42. Romano CL, Gala L, Logoluso N, et al. Septic shoulder prosthesis: Surgical treatment and results of a consecutive series of 20 cases. J Orthop Traumatol 2011; 12: S28-S29.
43. Sabesan VJ, Ho JC, Kovacevic D, et al. Two-stage reimplantation for treating prosthetic shoulder infections. Clin Orthop Relat Res 2011; 469: 2538-2543.

44. Seitz Jr WH and Damacen H. Staged exchange arthroplasty for shoulder sepsis. J Arthroplasty 2001; 17: 36-40.

45. Singh JA, Sperling JW, Schleck C, et al. Periprosthetic infections after shoulder hemiarthroplasty. J Shoulder Elbow Surg 2012; 21: 1304-1309.

46. Singh JA, Sperling JW, Schleck C, et al. Periprosthetic infections after total shoulder arthroplasty: a 33-year perspective. J Shoulder Elbow Surg 2012; 21: 1534-1541.

47. Stine IA, Lee B, Zalavras CG, et al. Management of chronic shoulder infections utilizing a fixed articulating antibiotic-loaded spacer. J Shoulder Elbow Surg 2010; 19: 739-748.

48. Strickland JP, Sperling JW and Cofield RH. The results of two-stage re-implantation for infected shoulder replacement. J Bone Joint Surg Br 2008; 90: 460-465.

49. Themistocleous G, Zalavras C, Stine I, et al. Prolonged implantation of an antibiotic cement spacer for management of shoulder sepsis in compromised patients. J Shoulder Elbow Surg 2007; 16: 701-705.

50. Twiss TJ and Crosby LA. Treatment of the infected total shoulder arthroplasty with antibiotic-impregnated cement spacers. Semin Arthroplasty 2010; 21: 204-208.

51. Wang B, Toye B, Desjardins M, et al. A 7-year retrospective review from 2005 to 2011 of Propionibacterium acnes shoulder infections in Ottawa, Ontario, Canada. Diagn Microbiol Infect Dis 2013; 75: 195-199.

52. Yian E, Chan PH, Navarro RA, et al. Surgeon-controllable risk factors for periprosthetic infection: an analysis of 8,056 shoulder arthroplasties. J Shoulder Elbow Surg 2017; 26: e160.

53. Zavala JA, Clark JC, Kissenberth MJ, et al. Management of deep infection after reverse total shoulder arthroplasty: a case series. J Shoulder Elbow Surg 2012; 21: 1310-1315.

54. Bala A, Penrose CT, Visgauss JD, et al. Total shoulder arthroplasty in patients with HIV infection: complications, comorbidities, and trends. J Shoulder Elbow Surg 2016; 25: 1971-1979.

55. Trappey GJt, O'Connor DP and Edwards TB. What are the instability and infection rates after reverse shoulder arthroplasty? Clin Orthop Relat Res 2010; 469: 2505-2511.

56. Magnan B, Bondi M, Vecchini E, et al. A preformed antibiotic-loaded spacer for treatment for septic arthritis of the shoulder. Musculoskelet Surg 2014; 98: 15-20.

57. Anakwenze O, Fokin A, Chocas M, et al. Complications in total shoulder and reverse total shoulder arthroplasty by body mass index. J Shoulder Elbow Surg 2017; 30: 30.

58. Coste JS, Reig S, Trojani C, et al. The management of infection in arthroplasty of the shoulder. J Bone Joint Surg Br 2004; 86: 69.

59. Everhart JS, Bishop JY and Barlow JD. Medical comorbidities and perioperative allogeneic red blood cell transfusion are risk factors for surgical site infection after shoulder arthroplasty. J Shoulder Elbow Surg 2017; 26: 1922-1930. 
60. Florschutz AV, Lane PD and Crosby LA. Infection after primary anatomic versus primary reverse total shoulder arthroplasty. J Shoulder Elbow Surg 2015; 24: 1296-1301.

61. Ghijselings S, Stuyck J and Debeer Prof P. Surgical treatment algorithm for infected shoulder arthroplasty: a retrospective analysis of 17 cases. Acta Orthop Belg 2013; 79: 626-635.

62. Hatta T, Werthel JD, Wagner ER, et al. Effect of smoking on complications following primary shoulder arthroplasty. J Shoulder Elbow Surg 2017; 26: 1-6.

63. Hou C, Gupta A, Chen M, et al. How do revised shoulders that are culture positive for Propionibacterium differ from those that are not? J Shoulder Elbow Surg 2015; 24 : 1427-1432.

64. Jacquot A, Sirveaux F, Roche O, et al. Surgical management of the infected reversed shoulder arthroplasty: a French multicenter study of reoperation in 32 patients. J Shoulder Elbow Surg 2015; 24: 1713-1722.

65. Jerosch J and Schneppenheim M. Management of infected shoulder replacement. Arch Orthop Trauma Surg 2003; 123: 209-214.

66. Lorenzetti AJ, Wongworawat MD, Jobe CM, et al. Cyanoacrylate microbial sealant may reduce the prevalence of positive cultures in revision shoulder arthroplasty. Clin Orthop Rel Res 2013; 471: 3225-3229.

67. Lovallo J, Helming J, Jafari SM, et al. Intraoperative intra-articular injection of gentamicin: will it decrease the risk of infection in total shoulder arthroplasty? J Shoulder Elbow Surg 2014; 23: 1272-1276.

68. Maccioni CB, Woodbridge AB, Balestro JC, et al. Low rate of Propionibacterium acnes in arthritic shoulders undergoing primary total shoulder replacement surgery using a strict specimen collection technique. J Shoulder Elbow Surg 2015; 24: 1206-1211.

69. Morris BJ, O'Connor DP, Torres D, et al. Risk factors for periprosthetic infection after reverse shoulder arthroplasty. J Shoulder Elbow Surg 2015; 24: 161-166.

70. Nowinski RJ, Gillespie RJ, Shishani Y, et al. Antibioticloaded bone cement reduces deep infection rates for primary reverse total shoulder arthroplasty: a retrospective, cohort study of 501 shoulders. J Shoulder Elbow Surg 2012; 21: 324-328.

71. Ortmaier R, Resch H, Hitzl W, et al. Treatment strategies for infection after reverse shoulder arthroplasty. Eur $J$ Orthop Surg Traumatol 2014; 24: 723-731.

72. Romano CL, Borens O, Monti L, et al. What treatment for periprosthetic shoulder infection? Results from a multicentre retrospective series. Int Orthop 2012; 36: 1011-1017.

73. Sperling JW, Hawkins RJ, Walch G, et al. Complications in total shoulder arthroplasty. J Bone Joint Surg Am 2001; 95: 563-569.

74. Verhelst L, Stuyck J, Bellemans J, et al. Resection arthroplasty of the shoulder as a salvage procedure for deep shoulder infection: does the use of a cement spacer improve outcome? J Shoulder Elbow Surg 2011; 20: 1224-1233.

75. Walch G, Bacle G, Ladermann A, et al. Do the indications, results, and complications of reverse shoulder arthroplasty change with surgeon's experience? J Shoulder Elbow Surg 2012; 21: 1470-1477.

76. Weber P, Utzschneider S, Sadoghi P, et al. Management of the infected shoulder prosthesis: a retrospective analysis and review of the literature. Int Orthop 2010; 35: 365-373.

77. Werner BC, Cancienne JM, Burrus MT, et al. The timing of elective shoulder surgery after shoulder injection affects postoperative infection risk in Medicare patients. J Shoulder Elbow Surg 2016; 25: 390-397.

78. Werthel JD, Hatta T, Schoch B, et al. Is previous nonarthroplasty surgery a risk factor for periprosthetic infection in primary shoulder arthroplasty? J Shoulder Elbow Surg 2017; 26: 635-640.

79. Zhang AL, Feeley BT, Schwartz BS, et al. Management of deep postoperative shoulder infections: is there a role for open biopsy during staged treatment? J Shoulder Elbow Surg 2015; 24: e15-e20.

80. Dilisio MF, Miller LR, Warner JJP, et al. Arthroscopic tissue culture for the evaluation of periprosthetic shoulder infection. J Bone Joint Surg Am 2014; 96: 1952-1958.

81. Frangiamore SJ, Saleh A, Grosso MJ, et al. Neer Award 2015: analysis of cytokine profiles in the diagnosis of periprosthetic joint infections of the shoulder. J Shoulder Elbow Surg 2017; 26: 186-196.

82. Frangiamore SJ, Saleh A, Grosso MJ, et al. Alphadefensin as a predictor of periprosthetic shoulder infection. J Shoulder Elbow Surg 2015; 24: 1021-1027.

83. Frangiamore SJ, Saleh A, Kovac MF, et al. Synovial fluid interleukin-6 as a predictor of periprosthetic shoulder infection. J Bone Joint Surg Am 2015; 97: 63-70.

84. Grosso MJ, Frangiamore SJ, Ricchetti ET, et al. Sensitivity of frozen section histology for identifying Propionibacterium acnes infections in revision shoulder arthroplasty. J Bone Joint Surg Am 2014; 96: 442-447.

85. Grosso MJ, Frangiamore SJ, Saleh A, et al. Poor utility of serum interleukin-6 levels to predict indolent periprosthetic shoulder infections. J Shoulder Elbow Surg 2014; 23: 1277-1281.

86. Marmor S, Bauer T, Desplaces N, et al. Multiplex antibody detection for noninvasive genus-level diagnosis of prosthetic joint infection. J Clin Microbiol 2016; 54: 1065-1073.

87. Nelson GN, Paxton ES, Narzikul A, et al. Leukocyte esterase in the diagnosis of shoulder periprosthetic joint infection. J Shoulder Elbow Surg 2015; 24: 1421-1426.

88. Piper KE, Fernandez-Sampedro M, Steckelberg KE, et al. C-reactive protein, erythrocyte sedimentation rate and orthopedic implant infection. PLoS One 2010; 5: e9358.

89. Piper KE, Jacobson MJ, Cofield RH, et al. Microbiologic diagnosis of prosthetic shoulder infection by use of implant sonication. J Clin Microbiol 2009; 47: 1878-1884.

90. Tashjian RZ, Granger EK and Zhang Y. Utility of prerevision tissue biopsy sample to predict revision shoulder arthroplasty culture results in at-risk patients. $J$ Shoulder Elbow Surg 2017; 26: 197-203. 
91. Villacis D, Merriman JA, Yalamanchili R, et al. Serum interleukin-6 as a marker of periprosthetic shoulder infection. J Bone Joint Surg Am 2014; 96: 41-45.

92. Crane JK, Hohman DW, Nodzo SR, et al. Antimicrobial susceptibility of Propionibacterium acnes isolates from shoulder surgery. Antimicrob Agents Chemother 2013; 57: $3424-3426$.

93. Nodzo SR, Hohman DW, Crane JK, et al. Hemolysis as a clinical marker for Propionibacterium acnes orthopedic infection. Am J Orthop 2014; 43: E93-E97.

94. Smucny M, Menendez ME, Ring D, et al. Inpatient surgical site infection after shoulder arthroplasty. J Shoulder Elbow Surg 2015; 24: 747-753.

95. Hatch MD, Daniels SD, Glerum KM, et al. The cost-effectiveness of vancomycin for preventing infections following shoulder arthroplasty: a break even analysis. J Shoulder Elbow Surg 2017; 26: e144-e145.

96. Sperling JW, Kozak TK, Hanssen AD, et al. Infection after shoulder arthroplasty. Clin Orthop Relat Res 2001; 382: 206-216.
97. Farshad $M$ and Gerber C. Reverse total shoulder arthroplasty - from the most to the least common complication. Int Orthop 2010; 34: 1075-1082.

98. Maier GS, Horas K, Seeger JB, et al. Is there an association between periprosthetic joint infection and low vitamin D levels? Int Orthop 2014; 38: 1499-1504.

99. Pinder EM, Ong JC, Bale RS, et al. Ten questions on prosthetic shoulder infection. Shoulder Elbow 2016; 8: 151-157.

100. Zimmerli W, Trampuz A and Ochsner PE. Prostheticjoint infections. $N$ Engl J Med 2004; 351: 1645-1654.

101. Trampuz A and Zimmerli W. Prosthetic joint infections: update in diagnosis and treatment. Swiss Med Wkly 2005; 135: 243-251.

102. Corvec Sp, Portillo MaE, Pasticci BM, et al. Epidemiology and new developments in the diagnosis of prosthetic joint infection. Int J Artif Organs 2012; 35: 923-934. 